\title{
Sensing Human Activities With Resonant Tuning
}

Ivan Poupyrev ${ }^{1}$

ivan.poupyrev@disneyresearch.com

Zhiquan Yeo ${ }^{1,2}$

zhiquan@disneyresearch.com

Josh Griffin ${ }^{1}$

joshdgriffin@disneyresearch.com

Scott Hudson ${ }^{2}$

scott.hudson@cs.cmu.edu

${ }^{1}$ Disney Research, Pittsburgh

4615 Forbes Avenue, Suit 420

Pittsburgh, PA 15213 USA

${ }^{2}$ HCI Institute,

School of Computer Science,

Carnegie Mellon University,

5000 Forbes Avenue,

Pittsburgh, PA 15213

Copyright is held by the author/owner(s).

CHI 2010, April 10-15, 2010, Atlanta, Georgia, USA.

ACM 978-1-60558-930-5/10/04.

\begin{abstract}
Designing new interactive experiences requires effective methods for sensing human activities. In this paper we propose new sensor architecture based on tracking changes in resonant frequency of objects that users are interacting with.
\end{abstract}

\section{Keywords}

sensors, embodied interfaces, resonant frequency.

\section{ACM Classification Keywords}

H5.2 Information interfaces and presentation: User Interfaces --- Input devices and strategies; B.4.2 Input/output and data communication: Input/Output Devices --- Channels and controllers.

\section{Introduction}

Development of new technologies for sensing human activities has profound impact on user interface design. They enable new styles of interactions with digital devices, machines and environments; they inspire new applications and products and, in some cases, launch whole new areas of HCI research and development. That is why inventing new sensing technologies, exploring their interactive properties and applications is one of the most important research directions in the field of human-computer interaction [1] 
This paper presents the development of a novel method for sensing human activities. It is based on continuous tracking of changes in electromagnetic resonant properties of conductive objects that happen due to interaction between human and those objects. In other words, by measuring how the electromagnetic resonant frequency changes when user does "something" with an object we can understand what was that "something" that the user has done.

Although fundamental physical principles behind our development are well known, they have not been exploited yet in designing sensors for human computer interaction. This is, perhaps, due to high computational cost that had made such sensors unfeasible until now. The proposed sensing approach, however, has potentially exciting properties, for example it allows to turn any conductive object into a touch-sensitive device that can detect both touch and the area of touch; it allows to "tag" objects and then track changes in their internal configuration; it can measure very small stretching and bending of twisted wires, which allows to design new types of interactive fabrics and smart materials. At the same time the resulted sensors are simple and reliable, they do not require instrumenting the user and can be connected to any object with a single wire. They provide very high resolution and sensitivity; they are selfcalibrating and resistant to electrical noise. We believe that proposed sensing architecture has wide range of uses from making new type of touch-sensitive and embodied interfaces to developing new interactive materials for wearable and ubiquitous computing.

\section{Background and Related Work}

Sensor is a transducer device that converts physical stimulus, such as light or motion, into signal that can be measured. In user interfaces humans supply stimu- lus to sensors that can be located in input devices, worn by the user, embedded into objects or environments. The details of the interface implementations strongly depend on what physical phenomenon is measured by sensors, e.g. resistance is used in knobs and resistive touch panels, light intensity are used in cameras, the direction and intensity of magnetic fields is used in proximity sensors (e.g. [3][4]), acceleration is used in shake sensing, amount of electrical charge is used in multi-touch capacitive input devices [5].

We propose to use yet another physical phenomena in designing user interfaces, a frequency of electromagnetic resonance. Electromagnetic resonance has been widely used in tuning, filter and oscillator circuits in radio communication, as well as in designing tagging and identification devices [2]. These devices, however, assume that resonant characteristics are known a priori and the user then simply manipulates a specific tag or identification card.

We, on the other hand, view any system of conductive objects as part of large resonant circuit that also includes humans. If we assume that the electrical properties of objects do not change, then any changes in the resonant properties of the system can happen only because of the user actions. Thus, we can identify human activities by tracking changes in resonant characteristics of the entire system. We are not aware of previous research in HCI that attempted to design user interfaces based on this approach to sensing.

\section{Sensing by Resonance}

The overall principles of sensing are presented on Figure 1. In this section we discuss the details of sensor design and its basic properties. 


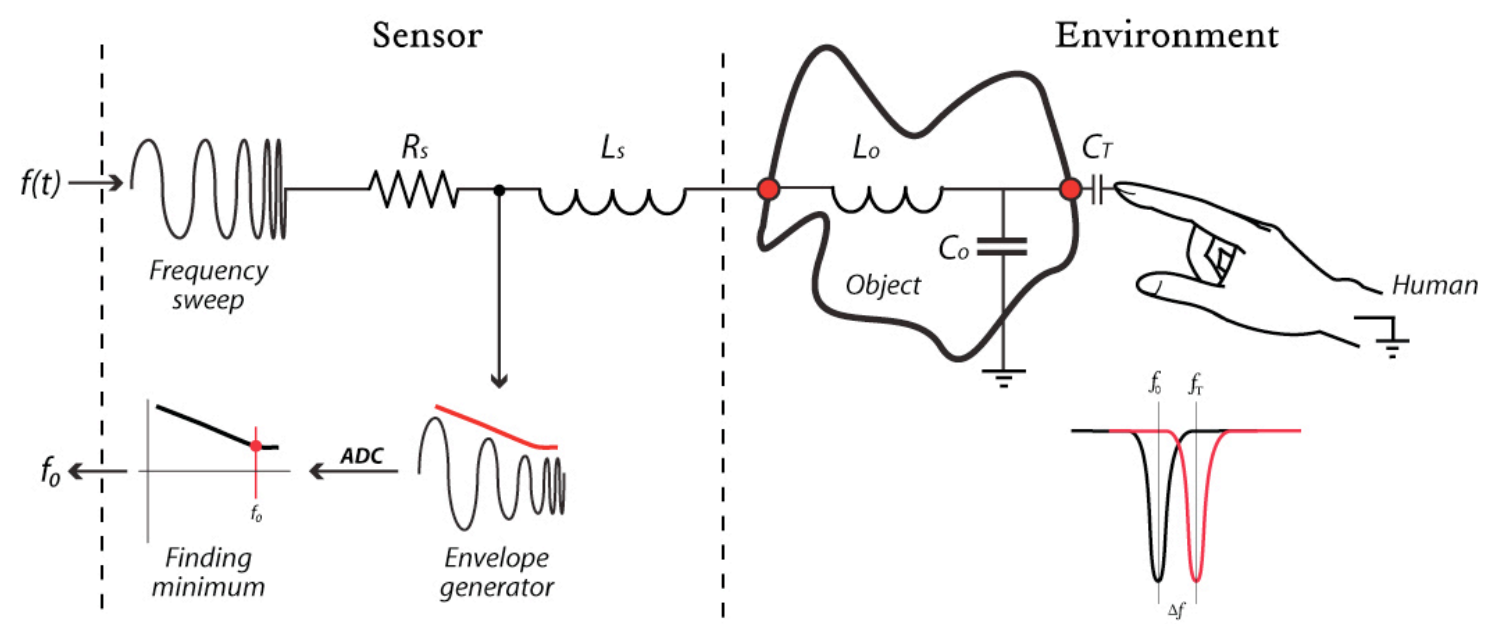

Fiaure 1: Basic architecture of the resonant tunina sensor

Physical principles

We can illustrate the basic idea behind sensor design using the following familiar example. A slight strike on a crystal glass would produce a characteristic ringing sound: a glass would vibrate at its resonant frequency. A crack in the glass material or water poured into the glass would change its resonant frequency and pitch of the sound. Therefore, we can estimate the internal state of the glass only by measuring the sound pitch when the glass is vibrating at its resonant frequency.

Similarly to crystal glass, any electrical system that includes capacitive and inductive elements would have a unique frequency at which alternating current floating through the system would oscillate, i.e. resonant frequency. Just like with crystal glass we can "strike" our system with an impulse signal, and then estimate it's resonant frequency by measuring the frequency of ripples in system response. A more practical approach is to force electrical oscillations by exciting system with periodic electrical signal at different frequencies, e.g. by performing a frequency sweep. At the resonant frequency the amplitude of alternating current reaches its maximum ${ }^{1}$. The resonant frequency can be also calculated as follows:

$$
f_{0}=\frac{1}{2 \pi \sqrt{L C}},(\text { Eq. } 1)
$$

here $L$ is inductance and $C$ is capacitance which together define reactive properties of the system.

Similarly to the example with crystal glass, any alternations or interactions that affect these reactive properties would change the resonance frequency of the total system. If we assume that all properties of objects in the system do not change then any changes in resonant frequency are because of the user interacting with the system. Therefore, we can infer user actions by

\footnotetext{
${ }^{1}$ We consider only serial resonant circuits.
} 


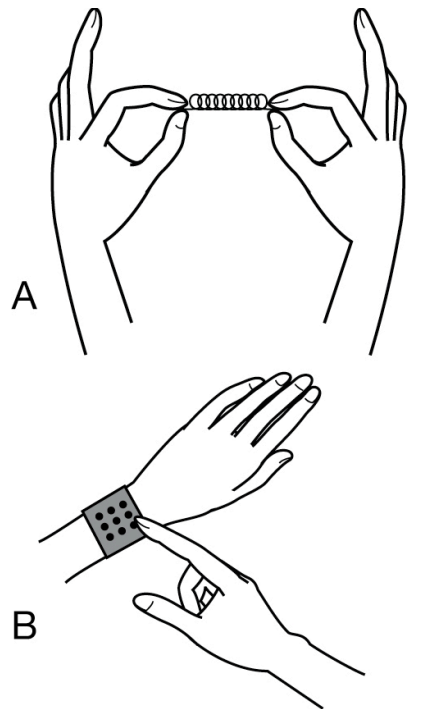

Figure 2: Categories of interactions a) Inductive interactions: stretching and deformations b) Capacitive interactions: touching and rigid manipulations. measuring the changes in the overall resonant frequency of the system.

\section{What objects we can sense?}

Electromagnetic resonance requires both capacitive and inductive properties. What objects we can use in designing our sensing solutions?

As it happens, most real-world conductive objects have capacitances and inductances that exist naturally due to specifics of objects mechanical structure $\left(C_{0}\right.$ and $L_{0}$ on Figure 1). For example, chain links in the wristwatch bracelet would naturally form capacitive links and a pair of twisted copper wires would create an inductive element. For most objects these parasitic capacitances and inductances are very small and the resonant frequency is extremely high, approaching infinity (see equation 1). Nevertheless, it can be measured by using simple technique that we discuss later in the paper.

To conclude, we can use any electrically conductive objects that, first, have weak capacitive and/or inductive reactances and second, afford physical interactions that allow users to alter these reactances and change the resonant frequency.

User interactions, inferring user actions

We break user interactions with objects into two alternative groups: capacitive and inductive (Figure 2).

Capacitive interactions change capacitive properties of the system. For example, a user touching a conductive object, such as a button, forms a capacitive link to ground increasing overall capacitance and decreasing the resonant frequency. Another example of capacitive interactions is physical re-configuration of the object, such as opening or closing a drawer in a metal cabinet, which leads to changes in parasitic capacitances and changes its resonant frequency.

Inductive interactions change inductive properties of the overall system. For example, stretching a metal spring would change its inductive reactance, since coil inductance depends on the distance between its turns.

Both capacitive and inductive interactions change a single physical property: resonant frequency. To infer user actions from changes in single measurable parameter we must establish one-to-one correspondence between user actions and parameter changes. While this is a general design problem for each particular sensing configuration, in most basic scenario we need to be able to block the effect of either capacitive or inductive interactions. That would allow us to be sure that changes in resonant frequency are the result of changes in one but not both of them. For example, if we are measuring stretching of twisted wire, which is

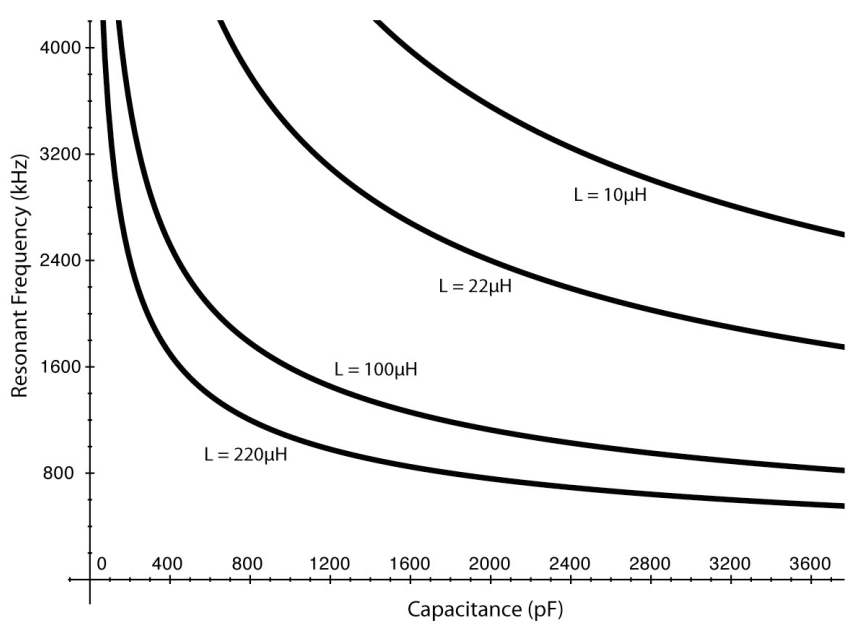

Figure 3: Resonant frequency versus capacitance for fixed values of inductance. 


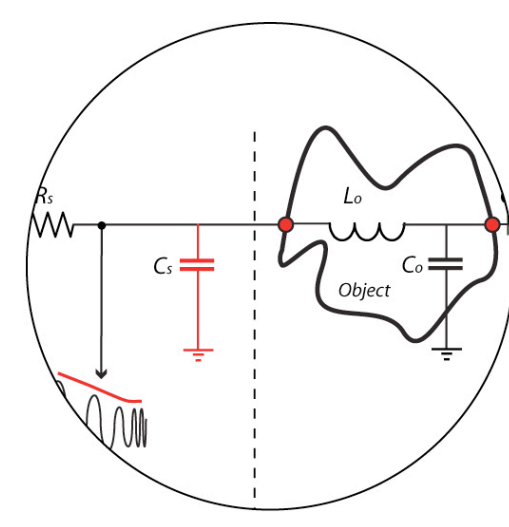

Figure 4: Adding capacitor $C_{s}$ would block influence of capacitive interactions.
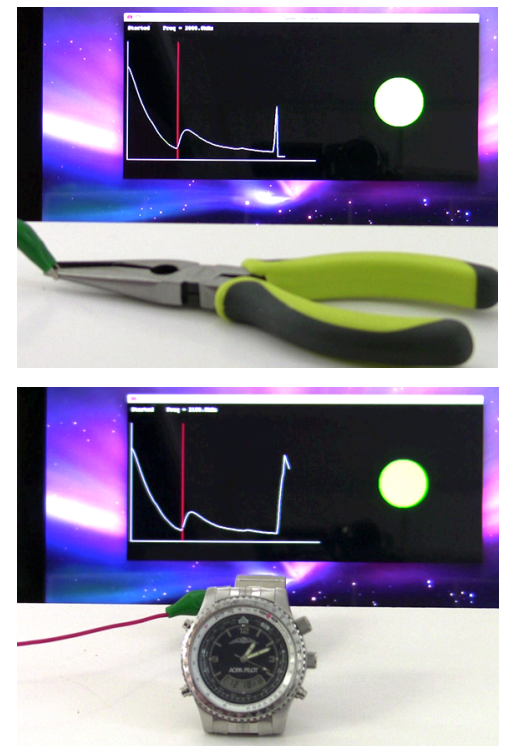

Figure 5: Measuring self-resonant frequency of everyday objects. inductive interaction, we would like to remove the influence of the user touching the wire, which is a capacitive interaction that would affect measurements.

Blocking the influence of alternative interaction can be accomplished by adding a biasing capacitor or inductor. As we can see from Figure 3, with the increase of capacitance the resonant frequency becomes less sensitive to small variations in capacitances, while sensitivity to changes in inductance is not affected, as long as inductance is very small. Therefore, by including large biasing capacitor we can effectively block the influence of capacitance (Figure 4). In example above, that would allow us to be sure that any changes in resonant frequency are coming from wire stretching only.

Measuring weak capacitances and inductances; resonance signatures of everyday conductive objects As we discussed above, object inductances and capacitances that we use for sensing are very low and, consequently, the corresponding resonant frequencies are very high. We can shift the resonant frequency into lower range by implicitly controlling one of the reactive parameters, e.g. either inductance or capacitance. Indeed, according to Figure 3, as we increase the inductance the resonant frequency shifts into lower range, e.g. below $5 \mathrm{Mhz}$, where it can be reliably measured even for very small capacitances. Therefore, adding a large biasing inductor $L_{s}$, as on Figure 1, allows accurate measurements of resonant frequencies for objects with very small capacitances. Similarly we can measure small inductances by using biasing capacitor (Figure 4).

Being able to measure the resonant frequencies for very small capacitances and inductances allows us to "tag" and recognize everyday objects by measuring and recording their unique resonance frequencies, e.g. self- resonant frequency. This allows for some interesting properties, such as self-calibration.

Self-calibration and sensitivity.

An interesting property of our sensing approach is that it allows for self-calibrating. Indeed, without the user interacting with objects their resonant frequency would be at self-resonance frequency. As the user is interacting with the system, the resonant frequency of the system would shift away from the self-resonance, e.g. in case of capacitive touch it would decrease, and after the user ceased interaction the resonant frequency returns to the initial state.

\section{Case Design: Making Everyday Objects Touch-Sensitive}

We have discussed the basic principles of designing sensing solutions based on measuring resonance. In this section we describe a case design for sensor that adds ad-hoc touch sensitivity to everyday objects.

The sensor continually tracks resonant frequency of any conductive object attached to it, such as a wristwatch or pliers as seen on Figure 5. In each tracking cycle we excite objects with a $500 \mathrm{~Hz}-3 \mathrm{MHz}$ frequency sweep. We use a biasing inductor $L_{s}=400 \mu \mathrm{H}$ (Figure 1 ) in order to shift objects' resonant frequencies into low range and block inductance effects. A small resistor $R_{s}$ is added to convert alternating current into voltage, which is then converted into time-varying DC signal using simple envelope-generator circuit. The signal is sampled using ADC convertor and the first local minimum is computed which would correspond to the resonant frequency of the current tracking cycle.

Figure 5 presents measurement of resonant frequency for pliers and watch. The real time graph of DC voltage 


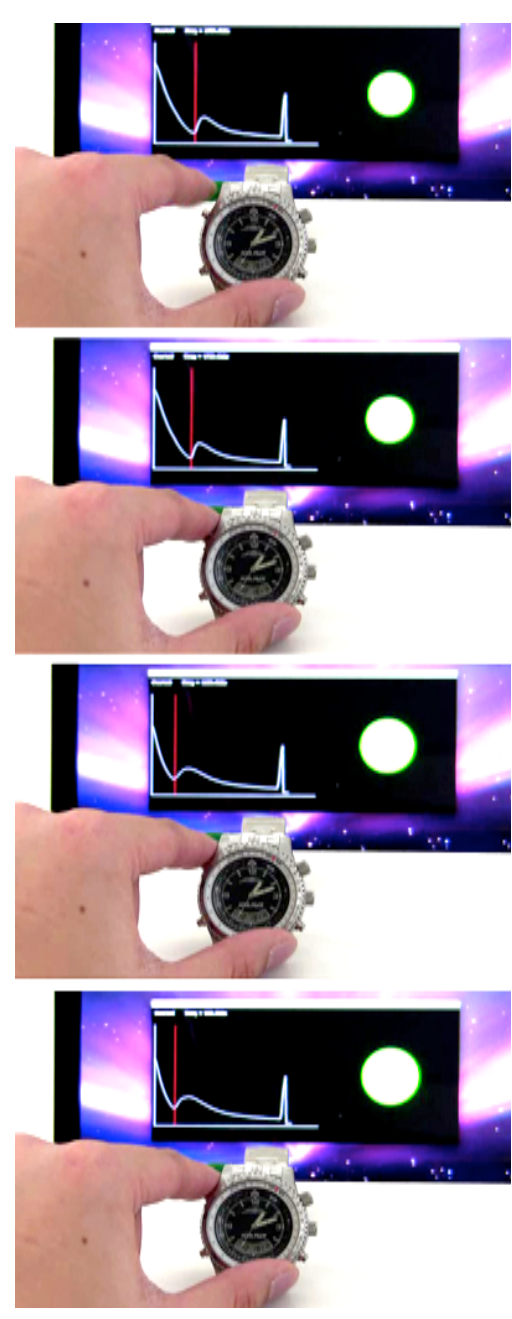

Figure 6: Wristwatch resonance frequency decreases when the user is touching it and the amount of shift depends on area of touch. versus frequency is behind objects; the red vertical line indicates objects resonant frequencies, which are $2 \mathrm{Mhz}$ and 2.1 Mhz for piers and watch correspondingly ${ }^{2}$.

When user is touching an object his finger forms a capacitive link, increasing overall capacitance and decreasing the resonant frequency. In fact, user finger forms a variable capacitor: as the he presses harder, the finger skin stretches increasing an area of touch and increasing capacitance. Figure 6 illustrates this phenomena: as the user presses harder, the vertical red line indicating current resonant frequency shifts from 2.1 Mhz, i.e. wristwatch self-resonant frequency, to $900 \mathrm{KHz}$. In the current implementation we sweep frequencies with $1 \mathrm{Khz}$ increment which gives us 100 distinct values from no touching to full touch condition, which is extremely sensitive.

This design demonstrates some of the interesting properties of our sensing approach: any conductive object can become touch-sensitive just by attaching a single wire, the sensing is analogue with high sensitivity to changes in area of touch, we can identify objects depending on their natural self-resonant frequency and consequently "tag" and recognize them later. A variety novel applications are possible even with this simple sensor that we designed just as a proof of concept.

\section{Future Work}

This paper presents our early investigation of new approach for sensing human activities and describes design of a novel touch sensor developed as a proof-ofconcept. This work is still in early stage with several

2 We should remind that these frequencies are adjusted with biasing inductor $L_{s}$, the actual resonant frequencies of these objects would be in gigahertz range. technological hurdlers that must to be overcome, e.g. reducing size of sensor, increasing update rate and improving precision. Investigating new sensing configurations implementing our approach is also very important direction of future work. This includes but not limited to designing sensors for stretching; investigating possibility for 2D touch-sensors and multi-touch, designing sensors for tracking changes in internal objects configuration and many more. The approach that we describe in this paper is universal in a sense that it proposes a different way of sensing human activities. We believe that it will lead to design of many new sensing solutions and exciting interactive experiences that were not possible before.

\section{References}

[1] Buxton, W. There's More to Interaction than Meets the Eye: Some Issues in Manual Input. In User Centered System Design: New Perspectives on HumanComputer Interaction. Lawrence Erlbaum Associates, 1986, pp. 319-337.

[2] Paradiso, J., Hsiao, K. Swept Frequency, Magnetically-Coupled Resonant Tags for Realtime, Continuous, Multiparameter Control in ACM CHI Extended Abstracts, 1999, pp. 212-213.

[3] Harrison, C., Hudson, S. Abracadabra: Wireless, High-Precision and Unpowered Finger Input for Very Small Mobile Devices. UIST 2009, pp. 121-124.

[4] Poupyrev, I., Oba, H., Ikeda, T., Iwabuchi, E. Designing Embodied Interfaces for Casual Sound Recording Devices. Extended Proceedings of CHI'08. 2008, Design Case Session: ACM: pp. 2129-2134

[5] Rekimoto, J. SmartSkin: An Infrastructure for Freehand Manipulation on Interactive Surfaces, CHI2002, 2002 